\title{
Factors Affecting Transfer of Training to the Workplace after Faculty Development Program: what do Trainers Think?
}

\author{
Muhammad Zafar Iqbal* and Mona Hmoud Al Sheikh \\ Department of Medical Education, Imam Abdulrahman Bin Faisal University, Saudi Arabia
}

Received: March 12, 2018; Published: April 03, 2018

*Corresponding author: Muhammad Zafar Iqbal, Department of medical education, Building 400, King Fahd University Hospital, Al-Khobar, Saudi Arabia, Tel: 966540761405; Email: drzafariqbal@live.com

\section{Abstract}

Introduction: Faculty development plays pivotal role in medical education if the institutes aim to develop competent and effective teachers. The eventual goal of faculty development is to transfer the newly acquired knowledge and skills to workplace to influence learning. If the training programs fail to foster transfer of training then the effectiveness of these training activities decreases significantly.

Objective: The current study aims to explore the factors that are involved in hindering or aiding the transfer of training (newly learned competencies) to workplace i.e., educational milieu.

Methods: This is cross-sectional qualitative study that has used purposive sampling technique. Seven program developers contributed in the study. The data was collected through semi-structured interviews, which was then analysed using thematic analysis. The interviews were commenced between October 2017 to January 2018.

Results: The interviews with program developers and faculty trainers revealed that transfer of training to educational practices is a complex and multidimensional process that is influenced by many variables which can categorized broadly into 3 groups: trainee characteristics, training design features and environmental factors.

Conclusion: The current study has implications for understanding the learning transfer after faculty development program and has provided a brief overview of the individual, programmatic and environmental factors that influence the transfer of training to educational setup. More research is required to understand the complexities of training transfer in detail, especially in the context of health sciences education.

Keywords: Transfer of training; Faculty development; Faculty training; Staff development; Teaching competence

\section{Introduction}

Medical teachers are reflective practitioners who make critical and responsible decisions within the educational field such as classrooms, clinics and the institutions. They cater students' individual characteristics, differences in their prior knowledge and their diverse learning styles that make the role of a teacher quite challenging in academia. The situation becomes more critical if the teachers are not well prepared to perform their diversified tasks and responsibilities. This shortcoming may sometimes be seen in new recruits and faculty members who haven't undergone through any structured teacher training rigor. This is specially a serious concern in medical education where health professionals are trained to be physicians and not the academic teachers [1]. In such circumstances, faculty development plays pivotal role in medical education if the institutes aim to develop competent and effective teachers [2]. Especially, it holds pivotal value when the institutes are undergoing through curricular reforms, restructuring and / or educational transformations [3].

Faculty development has gained increased popularity in the past decade in response to the innovative educational strategies in teaching, assessment and curriculum. Many medical organizations now offer a variety of activities to nurture their faculty and to equip them with essential teaching skills which can help them perform their diversified academic roles [4]. Faculty development is defined as a series of activities that strengthen and extend the existing knowledge, skill and attitude of the educators, leading to a change in their thinking, teaching practices and their educational 
behavior [5]. The tenacity of refining teaching practices is to support and maximize the learning process of students. In other words, the goal of faculty development is to transfer the newly acquired knowledge and skills to workplace to influence learning. The transfer of competence to workplace is defined as 'the effective (generalization) and continuing (maintenance) application in the job (educational) environment of the knowledge, skills and attitude gained in a faculty development context'[3].

However, transfer of learning to workplace is not a simple phenomenon and is easier said than done. An educator has to overcome numerous barriers, encountered in an educational environment, that hinder in translation of acquired skills to practice, leading to decreased transfer ratio. For instance, in a study, Ford et al [6]. Found out in existing literature that only $10 \%$ of the learning, resulting from FD activities, was transferred to the job. It is also worth mentioning that faculty development activities themselves are an important factor. These initiatives vary in goals, lengths, methods, targeted participants and so on. Henceforth, certain personal, professional and environmental variables do exist that interfere and affect the transfer process. The current literature highlights many factors which are involved in influencing the transfer regime in the context of higher education [7,8]. Nursing and human resource development but unfortunately not much work has been done in the field of medical education in finding out how transfer actually occurs and what factors are involved in it [9]. If we wish to improve the effectiveness of faculty development programs, then we need to know for sure that which variables really make a

Table 1: Description of participants. difference in the complex process of achieving transfer of learning. Without identifying and understanding these influencing variables, it will be quite challenging for the program developers to cater them while planning activities.

\section{Methods}

Transfer of training is a multi factorial process and involves many stakeholders (faculty developers, medical teachers, students and administrative bodies). All stakeholders involved in the process of faculty development have their own attributes and perceptions regarding transfer of training process. This cross sectional qualitative study aims to cater the perceptions of faculty developers in specific and aims to describe their view point's using their own words [10]. To achieve this goal, a purposive sampling was done and data was collected through semi-structured interviews, which were then analyzed using thematic analysis.

\section{Participants}

Nine medical educationists, who are actively involved in faculty development workshops and training programs in 5 health sciences institutes, were invited to contribute in the study. The invited medical educationists had 5 to 15 years of experience as health professional trainers in various faculty development workshops, symposia and structured degree programs. 7 out of 9 invites accepted the invitation and contributed in the study which was interviewed in detail. The details about the faculty developers, their experience, qualification and institutional affiliations are given in (Table 1).

\begin{tabular}{|c|c|c|c|c|}
\hline Interviewee & Profession & Experience as trainer & Qualification & Institution \\
\hline P1 & Medical Educationist & 18 & $\mathrm{PhD}$ & Islamic International University, Islamabad, Pakistan \\
\hline P2 & Medical Educationist & 15 & $\mathrm{PhD}$ & Islamic International University, Islamabad, Pakistan \\
\hline P3 & Medical Educationist & 14.5 & $\mathrm{PhD}$ & World Health Organization, Egypt \\
\hline P4 & Medical Educationist & 10 & $\mathrm{PhD}$ & $\begin{array}{c}\text { Imam Abdulrahman Bin Faisal University, Dammam, } \\
\text { Saudi Arabia }\end{array}$ \\
\hline P5 & Medical Educationist & 7 & PhD & Khyber Medical University, Peshawar, Pakistan \\
\hline P6 & Medical Educationist & 7 & PhD Scholar & Khyber Medical University, Peshawar, Pakistan \\
\hline P7 & Medical Educationist & 5 & PhD Scholar & University of Lahore, Pakistan \\
\hline
\end{tabular}

\section{Procedure}

All participants were interviewed by using open ended, semi-structured questions. The duration for each interview was approximately 30 minutes. The interviews took the form of a conversation where the participants responded to the questions (listed in Appendix A). All interviews were recorded and were transcribed by an independent data analyst. Post interview, all participants were sent a verbatim transcript of their interviews individually for verification of correct interpretation of their perspectives. All the participants were informed about the purpose of the study, method of interview recording, data collection and interpretation in the research project. All interviews were taken separately and therefore the identity of one interviewee over another was not disclosed. The interviews were commenced between October 2017 to January 2018.
Analysis

The thematic analysis was performed by both the authors by the procedure and protocols suggested by Longhofer et al [11]. To increase the objectivity of the analysis, the transcribed interviews were coded as numbers before starting the analysis. Both authors individually read all interviews and identified themes (notes) that were relevant to the research objectives. A constant comparative approach was used for data analysis.

\section{Results}

From the transcribed interviews, the identified noted were grouped into categories and from those categories, 3 main themes emerged.

a. Learner characteristics 
b. Program design characteristics

c. Educational environment characteristics

\section{Learner Characteristics}

Interviews with faculty developers revealed that individual characteristics of the faculty members who participate in faculty training play the most significant role in facilitating learning transfer. On probing the faculty developers further on the individual characteristics of learners, the prominent attributes found out were; cognitive abilities, motivation to learn and transfer, personal traits and the amount of experience.

\section{Cognitive Abilities}

"In my experience, faculty attending the workshops differs in their backgrounds and prior skillset. We try to cater all levels but unfortunately sometimes new recruits suffer as they are not familiar with the prior teaching concepts and principles" (P2). ".....of course, training activities prove to be very helpful for the teachers but only if they have prior knowledge and information regarding the concept being taught" (P4).The viewpoint of interviewees emphasizes on the issue that mostly the participants in any faculty training program come from varied backgrounds and have variable cognitive abilities. Most of the times, the newly taught information is easy for them to comprehend but sometimes, due to the difference in their cognitive abilities, it becomes challenging for the trainers to make them understand all essential concepts, leading to reduced retention and transfer.

\section{Motivation to Learn and Transfer}

"Faculty development is a rigorous process. If the participants are enthusiastic to attend, actively participate and learn, then they will certainly apply newly learned skills to their classrooms" (P1). "How can they learn something new if they are not internally motivated?" (P3). "I think it is not possible for the teachers to modify their existing teaching techniques if they are not internally driven" (P6).

\section{Personal Traits}

The personal attributes of the teacher are significant while translating the new skill to workplace. "If the faculty member is not confident in his/her capabilities then it will be difficult for him/her to deliver" (P3). "It is important that the faculty members recognise their training as a part of their lifelong learning process otherwise it will be difficult for them to internalize the new concepts and apply them" (P6). "Well, participants should be creative and active learners so that they can find new ways to teach their students" (P7).

\section{Amount of Experience}

"Experience has no parallel, even if the learned content is new, the prior teaching experience will always home handy" (P1). "I have seen that experienced faculty members adapt to the new concepts more quickly and try to implement it in their educational activities" (P3). However, this is not the case always as one of the interviewee expressed, "experienced teachers often show resistance to change.
Sometimes, it becomes difficult to make them understand that they have to change their teaching practices with time" (P5).

\section{Program Design Characteristics}

The second empirical feature influencing the transfer process is the program design, their duration, and structure and delivery methods. "We should try to make the training sessions as much hands-on as possible otherwise it will be just an interactive lecture. Participants usually lose their interest in such cases and don't try to implement later on" (P2). "Training programs should be designed which are practical. Usually clinical faculty doesn't have much time to take lengthy training courses. If the participants find utility and relevance of the program's content, they will be more enthusiastic and attentive" (P4). "While planning my training sessions, I try to be precise, realistic and practical so that participants can invest their precious time in gaining new knowledge, skills and attitude" (P6). "I think the programs should be well structured and transparent. In my experience, timely announcements, sharing reading content prior to training initiation and using implementation exercises within the workshops really motivate the faculty in learning and transferring process" (P7).

\section{Educational Environment Characteristics}

After personal and program attributes, third most significant characteristic is the educational and institutional environment were the faculty will be applying the newly acquired knowledge, skills and attributes. "It is important that the institutional and departmental hierarchy supports the faculty members in experimenting with new educational tools learned from faculty training" (P2). "Sometimes, the faculty do learn and show promising skills after faculty training but the environment is just not favorable for them to apply it" (P4). "Once they participate in faculty training, departmental support plays key role in implementation and transfer" (P5). "Sometimes, after the training, faculty feels reluctant in applying new teaching methods. They go back to their traditional methods. May be because there is no assessment method in most institutions to see if they are transferring the newly learned competencies?" (P6). "I think, peer support is extremely important, it gives them confidence and motivation to transfer the learning. Conversely, if the peer support is not there, the transfer process stops gradually" (P7)

\section{Discussion}

Faculty development programs aim to improve teaching competence and transfer of training to workplace. The effectiveness of faculty training reduces significantly if the trained teachers fail to implement new teaching and learning modalities [4]. However, transfer of training to educational practices is a complex and multidimensional process that takes place before, during and after training and involves many factors that aid and/or hinder in it [12]. In the current study, we have tried to find out these influencing variable that affect the transfer process through the perspectives of faculty trainers and program developers. After careful analysis, we have categorized these influencing factors into 3 groups: trainee characteristics, training design features, and work environmental factors. Similar categorization can be seen in the existing literature 
which are in synchronization with our findings [3,12]. Learners are the core component of any training activity and their attributes play key role in achieving competence and transfer.

Changing teaching practices and transferring new modalities require immense motivation, initiative and positivity and involve the investment of an individual's time, energy, and resources. The attributes which faculty developers think are empirical for the learning transfer are cognitive abilities, motivation to learn \& transfer, personal traits and the amount of experience. However, the list of learners' characteristics is not limited to it. Other attributes of the learner has also been observed in previous studies. For instance, leadership abilities, persistence, understanding systematic limitations, openness to change and risk taking [8]. These characteristics are often considered as hallmarks of academic leadership and effective teaching. However, the current study did not look into the factors that contribute to the intrinsic and extrinsic motivation of the learner, which is still a gap in the literature.

A variety of faculty development design elements exist that significantly influence transfer to learning, such as, the use of active learning strategies, cognitive load balancing, constructive feedback, hands-on training practice and the effective use of technology tools [13]. The faculty training activities vary drastically in their length and breadth, depending upon the institutional goals, dedication and resources. For instance, most training sessions consist of short, one or two days activity while some are longitudinal programs spread over weeks or months. We cannot simply assume that the level of competencies acquired in these varied programs would be the same. In health sciences, scarce literature is available on finding the effectiveness of these training programs [14]. In the available literature, the effectiveness of training and transfer intention is usually based on self-reported feedbacks by the faculty and they claim increased teaching effectiveness after faculty training but which intervention has maximum impact on the transfer process is still not well known and is least researched.

The training programs also differ in instructional designs, delivery methods and evaluation criteria. Most of the times, faculty training programs are designed on the wish list of the faculty members or administrative authorities which lead to focused training on desired skills and fail to cater generic teaching competencies [2]. It was also found out in this study that most training programs do not provide follow-up protocols and tend not to observe whether the intended outcomes have been achieved or not. This may be due to the lack of assessment policies within the faculty development programs. In future, research is required to design a systematic assessment process that can actually evaluate the teaching effectiveness and the extent of training transfer by incorporating third party reviewers i.e., peers, administrators, students and faculty developers instead of self-evaluation $[14,15]$.

Third domain that influences the training transfer process is the educational, departmental and/or institutional factors. Research shows that teachers are more likely to transfer their learning to their workplace when a supportive environment is in place [16].
The support in work environment can come from various levels and forms. Findings from the current study demonstrate that sources of support in trainees' work environments come from the institutions, departments, peers and students. Organizations can provide support through perceptions of a quality-driven culture and acknowledgement of innovative teaching practices. Departments can support their teachers through encouragement and recognition to try different teaching tools and modalities $[6,8]$. Rock advocated that an important contributing factor influencing transfer is the alignment between teachers' transfer efforts and institutional or departmental goals. If there is disharmony among the trainee and institutional goals or sufficient support system doesn't exist within the institution / department, then it will lead to discouragement and demonization of the teacher and eventually reduced transfer of training.

\section{Limitations}

The current study has incorporated the perceptions of the faculty trainers and does not provide insights on perceptions of other stakeholders such as faculty members themselves who participate in these training activities and the students who are at the receiving end of training transfer process. In addition to it, limited sample size was used for convenience purpose. In future a more extensive and in depth interview study should be conducted that might shed light on additional aspects involved in transfer of training process. Due to the limited number of study participants, the perspectives of the program developers may not necessarily be generalizable to training and development programs of other disciplines and professions.

\section{Conclusion}

The current study has implications for understanding the learning transfer after faculty development program and has provided a brief overview of the individual, programmatic and environmental factors that influence the transfer of training to educational setup. However, we advocate that more research is required to look into the process of training transfer in detail, especially in the context of health sciences education. A better understanding of transfer process and its attributes will help the program developers in designing more structured and evidencebased training programs that can ensure the achievement of teaching competence and transfer of training to workplace.

\section{References}

1. Steinert Y, Mann K, Centeno A, Dolmans D, Spencer J, et al. (2006) A systematic review of faculty development initiatives designed to improve teaching effectiveness in medical education: BEME Guide No. 8. Med Teach 28(6): 497-526.

2. Al Eraky MM, McLean M (2012) The Compass Model to plan faculty development programs. Med Educ Dev 2(1): 4.

3. Rijdt C De, Stes A, Vleuten C Van Der, Dochy F (2013) Influencing variables and moderators of transfer of learning to the workplace within the area of staff development in higher education: Research review. Educ Res Rev 8: 48-74.

4. Steinert Y, Mann K, Anderson B, Barnett BM, Centeno A, et al. (2016) A systematic review of faculty development initiatives designed to enhance teaching effectiveness: A 10-year update: BEME Guide No. 40 


$$
\text { 38(8): 769-786. }
$$

5. Fenstermacher GD, Berliner DC (1985) Determining the value of staff development. Elem Sch J 85(3): 281-314.

6. Ford JK, Yelon SL, Billington AQ (2011) How much is transferred from training to the job? The $10 \%$ delusion as a catalyst for thinking about transfer. Perform Improv Q 24(2): 7-24.

7. Ginns P, Kitay J, Prosse M (2010) Transfer of academic staff learning in a research-intensive university. Teach High Educ 15(3): 235-246.

8. Rock KZ (2014) Transferring learning from faculty development to the classroom. J Nurs Educ 53(12): 678-684.

9. Singh T, De Grave W, Ganjiwale J, Muijtjens A, van der Vleuten C (2014) Paying attention to intention to transfer in faculty development using the theory of planned behavior. Am J Educ Res 2(6): 361-365.

10. Lyberg-Åhlander V, Lundskog M, Hansson K (2013) Experiencing the role of PBL tutor. Clin Linguist Phon 28(1-2): 36-46.

11. Longhofer J, Floersch J, Hoy J (2012) Qualitative methods for practice research. Oxford University Press.

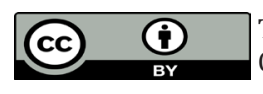

This work is licensed under Creative Commons Attribution 4.0 License

Submission Link: https://biomedres.us/submit-manuscript.php
12. Yelon SL, Ford JK, Anderson WA (2014) Twelve tips for increasing transfer of training from faculty development programs. Med Teach 36(11): 945-950.

13. Sommer J, Lanier C, Perron NJ, Nendaz M, Clavet D, et al. (2016) A teaching skills assessment tool inspired by the Calgary-Cambridge model and the patient-centered approach. Patient Educ Couns 99(4): 600-609.

14. Iqbal MZ, M Al Eraky M (2018) Using entrustable professional activities (EPAs) to assess teaching competence and transfer of training: A personal view. Med Teach 1-2.

15. Dewey CM, Jonker G, Ten Cate O, Turner TL (2017) Entrustable professional activities (EPAs) for teachers in medical education: Has the time come? Med Teach 39(8): 894-896.

16. Chiaburu DS, Dam K Van, Hutchins HM (2010) Social support in the workplace and training transfer : A longitudinal analysis. Int J Sel Assess 18(2): 187-200.

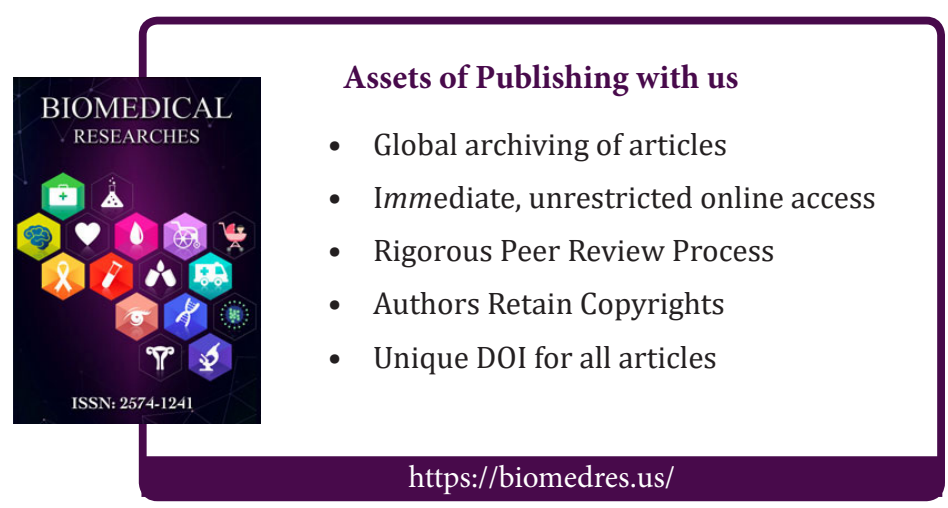

